Alexandra Popa

Holistic Dental \& Medical Institute of Bucharest - ROPOSTURO,

Bucharest, Romania

e-mail: alesandra17popa@gmail.com

\section{Essentials of \\ Dental Photography}

Author: Irfan Ahmad

Publisher:Wiley-Blackwell, Hoboken, NJ, USA

Language: English

ISBN: 978-1-119-31208-6

Edition: $1 / \mathrm{e}$

Publish Year: 2020

Pages: 360, illustrated

Price: $€ 79.10$

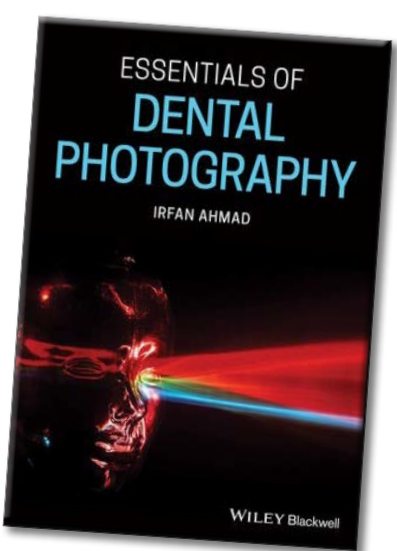

Photography have increasingly come to occupy an important place in medicine, in medical clinical specialties and a predominant role in dentistry.

Over time, a number of authors have been concerned with presenting their experience in photography, respectively in dental photography.

Dr. Irfan Ahmad, a member of the European Academy of Aesthetic Dentistry, author of numerous books on photography and dental aesthetics, presents over 10 chapters gathered in 3 sections detailing his experience in the book entitled "Essentials of dental photography".

Section 1, Equipment and Concepts presents the photographic equipment (cameras, lens, lighting and supports), dental armamentarium and clinical considerations, technical concepts and settings, composition (dominance, positioning, leading the eye, balance) and standardization.

Section 2, Photographic Set-ups describes extra and intra oral images, portraiture, bench images and special applications (detailed analysis of hard and soft tissue, color fidelity, shade analysis, scale reference markers, tooth whitening, phonetics, occlusal analysis and endodontic documentation).

Section 3, Processing Images discusses image processing (monitor calibration, imaging software, color spaces, white balance, orientation, scaling and cropping) and exporting, managing and using images.

Each chapter is richly illustrated and accompanied by significant recent references

"Essentials of dental photography" is a valuable guide for dental practice, useful for both dentists and dental assistants, dental technicians, but also young graduates for follow-up treatments, archiving information in the current activity and educating patients.

The Books Review is drafted in the reviewer's sole wording and illustrates his opinions. 